\title{
Haarausfall! Erhöhtes Prostatakrebsrisiko?
}

\author{
Anhand der Haarpracht eines Mannes lässt sich vermutlich sein Prostata- \\ karzinomrisiko abschätzen. Bei Männern mit Glatze ist demnach Vorsicht \\ geboten.
}

Estons

beginnt meist mit Geheimratsecken, die Stirn wird höher und schließlich lichtet sich das Haar am Scheitel. Gut $70 \%$ der Männer büßen im Laufe ihres Lebens einen Teil ihrer Haarpracht ein. Doch abgesehen von der ästhetischen Komponente birgt die androgenetische Alopezie handfeste gesundheitliche Risiken. Denn offenbar steht der Haarverlust für ein erhöhtes Erkrankungsrisiko der Prostata.

Ein derartiger Zusammenhang wird schon länger vermutet und ist bereits in mehreren Studien untersucht worden, allerdings mit inkonsistenten Ergebnissen. Urologen der Universitätsklinik in Chengdu/China wollten nun für mehr Klarheit sorgen und initiierten eine umfassende Metaanalyse. Insgesamt $17 \mathrm{Stu}-$ dien mit 68.448 Patienten erfüllten die
Auswahlkriterien und konnten in der Auswertung berücksichtigt werden. In zwölf der 17 Studien lag der Fokus auf dem Prostatakarzinom (PC), in fünf auf der benignen Prostatahyperplasie (BPH) und in einer waren PC und BPH Gegenstand des Interesses.

Tatsächlich stieg mit einer androgenetische Alopezie das Risiko der Männer, an einer aggressiven Form des Prostatakarzinoms oder einer benignen Prostatahyperplasie zu erkranken. Mit nichtaggressiven Formen des PC hingegen war die androgenetische Alopezie nicht assoziiert. Die Auswertung unter Berücksichtigung verschiedener Haarausfallmuster ergab, dass eine Tonsur mit einem erhöhten Prostatakrebsrisiko assoziiert war, aber nicht mit einem erhöhten BPH-Risiko. Auch für BPH und Ton- sur mit Stirnglatze fand sich kein statistisch relevanter Zusammenhang.

Männer mit Glatze haben ein höheres Risiko, eine benigne Prostatahyperplasie zu entwickeln oder an einem aggressiven PC zu erkranken, als Männer ohne Glatze, so das Resümee der Urologen aus China. Dass der Verlust der Haare als Indiz für ein erhöhtes Risiko für Prostataerkrankungen gelten kann, führen die Urologen auf ähnliche pathophysiologische Muster zurück. Bei Erkrankungen der Prostata wie auch beim anlagebedingten Haarausfall spielen Androgene und das Alter eine entscheidende Rolle. Belastbare Beweise gäbe es für diese Hypothese aber nicht, wie die Urologen betonen. Sie fordern daher, in weiteren Studien den Zusammenhang von Haarausfall und Prostataerkrankungen genauer zu beschreiben sowie die zugrunde liegenden pathologischen Mechanismen zu erforschen.

$(d k)$

Jin T et al. Association between male pattern baldness and prostate disease: A meta-analysis. Urol Oncol 2017, online 17. Oktober

\section{Ein Delir im Alter muss nicht sein}

\section{Jeder zweite geriatrische Patient erleidet im Krankenhaus ein Delir - oft unbemerkt. Doch mit regelmäßiger Nahrungs- und Flüssigkeitskontrolle, Stressvermeidung und kognitiver Stimulation lassen sich die meisten Delire vermeiden.}

U ntersuchungen legen nahe, dass jeder zweite geriatrische Patient, der in eine Klinik eingewiesen wird, dort ein Delir erleidet. Bei Patienten nach einer Op. liege die Prävalenz zwischen 10 und $60 \%$, erläuterte Prof. Michael Hüll, Emmendingen. Je älter die Patienten und je länger die Op., umso höher das Risiko. Aber auch in Pflegeheimen seien zu einem beliebigen Zeitpunkt 5-30\% der Bewohner betroffen.

Nicht immer sind die Symptome so ausgeprägt, dass sie von Ärzten und Pflegenden sofort erkannt werden. Nach Studiendaten wird etwa ein Drittel bis die Hälfte der Delire nicht bemerkt. Besonders hoch ist diese Gefahr bei Patienten mit psychischen Störungen: Hier gehen die Ärzte wohl häufig davon aus, dass die Delirsymptome durch die Erkrankung bedingt sind. „Bei einer psychiatrischen Vordiagnose ist die Wahrscheinlichkeit, dass ein Delir übersehen wird, dreifach erhöht", sagte Hüll.

Ein Delir zu erkennen und zu vermeiden sei jedoch für die Prognose der Betroffenen sehr wichtig. Der Psychiater erinnerte an die erhöhte Mortalität: ,Jeder Delirtag erhöht das Sterberisiko.“ Nach vier Tagen Delir sei die Sechs-Monats-Mortalität verdoppelt, nach sechs Tagen zweieinhalbfach höher als bei Patienten ohne Delir. Zur Diagnose empfiehlt Hüll einfache Instrumente wie die "Confusion Assesment Method (CAM)“. Dabei wird fremdanamnestisch abgefragt, ob sich der Zustand des Patienten akut verändert hat oder ob es deutliche

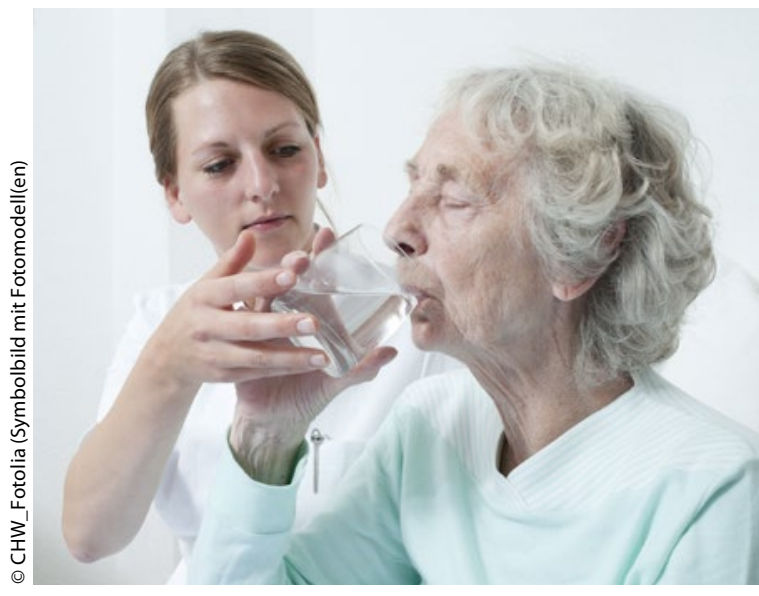

Tagesschwankungen gibt. Auch Konzentrationsprobleme, leichte Ablenkbarkeit, inkohärentes Denken sowie ein getrübter Bewusstseinszustand fließen in die Beurteilung ein. Maximal möglich sind vier Punkte, bei drei Punkten ist ein Delir sehr wahrscheinlich. Eine Variante ist die CAM-Severity-Skala (CAM-S), sie erfasst auch die Stärke der Aufmerksamkeitsprobleme, Denkstörungen und Be- 